\title{
Mujeres deportistas: poca visibilidad y mucho estereotipo
}

\section{Sportswomen: low visibility and largely stereotyped}

\section{Mulehres esportistas: baixa visibilidade e muito estereótipo}

\author{
Mujika-Alberdi, A. ${ }^{1}$, García-Arrizabalaga, I. ${ }^{2}$, Gibaja-Martíns, J.J. ${ }^{3}$ \\ ${ }^{1}$ Universidad de Deusto $;{ }^{2}$ Universidad de Deusto; ${ }^{3}$ Universidad de Deusto
}

* Trabajo de campo financiado gracias al reconocimiento otorgado por el Gobierno Vasco al equipo de investigación "Comunicación" IT1212-19 en su máxima categoría en el tramo 2019-2021.

\section{RESUMEN}

En el artículo se analiza la opinión ciudadana sobre la imagen de las mujeres deportistas en relación con la de los hombres deportistas en el Territorio Histórico de Gipuzkoa (País Vasco, España). En los últimos años, por una parte, se constata un avance significativo en la paridad de la práctica deportiva y, por otra parte, se observa un alto rendimiento deportivo de las mujeres deportistas que se ha hecho visible en la medida que han tenido la oportunidad de demostrarlo. Sin embargo, los medios de comunicación siguen ofreciendo a menudo una imagen estereotipada de las mujeres deportistas reproducida luego en la opinión pública. El artículo muestra los resultados de una encuesta realizada a la población guipuzcoana sobre la visibilidad/invisibilidad de las mujeres deportistas y los estereotipos asociados a su imagen. Los resultados indican que todavía, y a pesar de diferentes esfuerzos, las mujeres deportistas son poco visibles para la población. Tanto la notoriedad espontánea como la asistida de las mujeres deportistas es muy inferior a la de los hombres deportistas. La poca visibilidad se añade a otro problema, la perdurabilidad de los estereotipos. Se confirma la tendencia a incidir en cuestiones ligadas al aspecto físico, así como la atribución de diferentes valores según el sexo del deportista. A pesar de todo, se ha identificado una predisposición favorable hacia el consumo de deporte practicado por mujeres. En definitiva, se observan algunos pasos en la dirección hacia la paridad, pero el reto sigue aún vigente

Palabras clave: sociología del deporte; encuesta de opinión; sesgo de género; estereotipo.

\section{ABSTRACT}

The article analyzes the public opinion on the image of sportswomen in relation to that of sportsmen in the Historical Territory of Gipuzkoa (Basque Country, Spain). In recent years, on the one hand, there has been a significant advance in the parity of sports practice and, on the other hand, a high sports performance of female athletes has been observed, which has become visible to the extent that they have had the opportunity to prove it. However, the media often continue to offer a stereotypical image of female athletes later reproduced in public opinion. The article shows the results of a survey carried out among the population of Gipuzkoa on the visibility / invisibility of sportswomen and the stereotypes associated with their image. The results indicate that still, and despite different efforts, female athletes are not very visible to the population. Both the spontaneous and assisted notoriety of female athletes is much lower 


\section{Mujika-Alberdi, A.; García-Arrizabalaga, I.; Gibaja-Martíns, J. J.}

than that of male athletes. Low visibility adds to another problem, the persistence of stereotypes. The tendency to influence issues related to physical appearance is confirmed, as well as the attribution of different values according to the athlete's sex. Despite everything, a favorable predisposition towards the consumption of sports practiced by women has been identified. In short, there are some steps towards parity, but the challenge remains

Keywords: sport sociology; opinion survey; gender bias; stereotype.

\section{RESUMO}

O artigo analisa a opinião pública sobre a imagem das esportistas mulehres em relação à dos esportistas homens no Território Histórico de Gipuzkoa (País Basco, Espanha). Nos últimos anos, por um lado, houve um avanço significativo na paridade da prática esportiva e, por outro lado, observou-se um alto desempenho esportivo das atletas femininas, que se tornou visível na medida em que tiveram o oportunidade de provar isso. No entanto, a mídia muitas vezes continua a oferecer uma imagem estereotipada de atletas femininas posteriormente reproduzida na opinião pública. $\mathrm{O}$ artigo apresenta os resultados de uma pesquisa realizada junto à população gipuzkoana sobre a visibilidade / invisibilidade das esportistas e os estereótipos associados à sua imagem. Os resultados indicam que ainda, e apesar dos esforços diferenciados, as atletas femininas não são muito visíveis para a população. Tanto a notoriedade espontânea quanto a assistida de atletas do sexo feminino é muito menor do que a dos atletas do sexo masculino. A baixa visibilidade aumenta outro problema, a persistência de estereótipos. Confirma-se a tendência de influenciar questões relacionadas à aparência física, bem como a atribuição de valores diferenciados de acordo com o sexo do atleta. Apesar de tudo, identificou-se uma predisposição favorável ao consumo de esportes praticados por mulheres. Em suma, existem alguns passos em direção à paridade, mas o desafio ainda está em vigor.

Palavras chave: sociologia do esporte; pesquisa de opiniao: preconceito de genero; estereótipo.

\section{INTRODUCCIÓN}

Según la última Encuesta de Hábitos Deportivos en España (2015), el 46,2\% de la población española practica actividades físico-deportivas al menos una vez por semana, lo que supone un incremento de 12,6 puntos porcentuales respecto a la medición anterior, realizada en 2010 (33,6\%). Si analizamos este incremento según la variable sexo, en el caso de las mujeres el incremento en la práctica físico-deportiva (al menos una vez por semana) es aún mayor, pues ha pasado del $28,8 \%$ en 2010 al 42,1\% en $2015(13,3$ puntos porcentuales de incremento). En el caso de los hombres el porcentaje ha subido 4,9 puntos porcentuales (de 45,5\% en 2010 a 50,4\% en 2015).

En el ámbito del deporte federado, el Anuario de Estadísticas Deportivas (2019) informa de que en España había en 2010 algo más de 3,5 millones de licencias federativas, de las cuales algo más del $80 \%$ eran hombres y cerca del $20 \%$ mujeres. En 2015, el número de fichas federativas apenas sufre alguna modificación, y el porcentaje de hombres se reduce al $78,9 \%$ y el de las mujeres aumenta hasta el $21,5 \%$. Ya en 2018, con un total de 3.866 .867 deportistas federados en 66 disciplinas diferentes, el porcentaje de mujeres asciende hasta el 23\% . En esta misma línea, y según la misma fuente, el número de Deportistas de Alto Nivel (DAN ) era de 3.212 deportistas en 2010, donde algo más del $66 \%$ eran hombres y casi un $34 \%$ mujeres. En 2015, sobre un total 3.893 eran hombres el $65,4 \%$ y mujeres el $34,6 \%$. El último dato de la serie que ofrece esta fuente, el de 2018, eleva a 4.962 los deportistas DAN, donde el porcentaje de hombres es del $62,8 \%$ y el de mujeres $37,2 \%$. En resumen, toda esta batería de datos sugiere una tendencia hacia la reducción de la brecha de género en la práctica deportiva en los distintos niveles deportivos, pero también la existencia de una doble velocidad si se compara la actividad física deportiva y el deporte competitivo.

El Anuario de Estadísticas Deportivas (2019) aporta otros datos interesantes como el rendimiento de los deportistas. Por ejemplo, se observa que el número de récords de España alcanzados en 2018 para el total de categorías asciende a 473, de los cuales, $242(51,2 \%)$ son récords de España obtenidos por mujeres deportistas. Este dato sugiere, a falta de indicadores más precisos, que el alto rendimiento deportivo de las mujeres deportistas se hace visible en la medida que han tenido oportunidad de demostrarlo. 


\section{Visibilidad social de las mujeres deportistas}

La Tabla 1 recoge los datos ofrecidos por el Comité Olímpico Español (www.coe.es) sobre participación femenina, así como las medallas obtenidas en los Juegos Olímpicos. Se observa que, en Barcelona 92, el $29,3 \%$ de los deportistas españoles olímpicos fue mujer, mientras que en Rio ascendió hasta el 46,8\%. En cuanto al medallero de los representantes españoles, en Barcelona 92, el 36,4\% del total de medallas fueron obtenidas por mujeres y, en Rio 2016, este porcentaje ascendió a 52,9\%. Los datos muestran el aumento de la participación de las mujeres en la élite deportiva y también el alto rendimiento de las deportistas.

Tabla 1. La representación femenina española en los Juegos Olímpicos y medallero

\begin{tabular}{|c|c|c|c|c|c|c|}
\hline $\begin{array}{c}\text { Olimpi } \\
\text { adas }\end{array}$ & $\begin{array}{l}\text { Núme } \\
\text { ro de } \\
\text { deport } \\
\text { istas } \\
\text { homb } \\
\text { res }\end{array}$ & $\begin{array}{l}\text { Núme } \\
\text { ro de } \\
\text { deport } \\
\text { istas } \\
\text { mujer } \\
\text { es }\end{array}$ & $\begin{array}{c}\% \\
\text { mujer } \\
\text { es } \\
\text { deport } \\
\text { istas }\end{array}$ & $\begin{array}{c}\text { Medal } \\
\text { las } \\
\text { obteni } \\
\text { das } \\
\text { por } \\
\text { deport } \\
\text { istas } \\
\text { homb } \\
\text { res }\end{array}$ & $\begin{array}{c}\text { Medal } \\
\text { las } \\
\text { obteni } \\
\text { das } \\
\text { por } \\
\text { deport } \\
\text { istas } \\
\text { mujer } \\
\text { es }\end{array}$ & $\begin{array}{c}\% \\
\text { medal } \\
\text { las } \\
\text { obteni } \\
\text { das } \\
\text { por } \\
\text { mujer } \\
\text { es } \\
\text { deport } \\
\text { istas }\end{array}$ \\
\hline $\begin{array}{l}\text { Barcel } \\
\text { ona } \\
1992\end{array}$ & 302 & 125 & $29,3 \%$ & 14 & 8 & $36,4 \%$ \\
\hline $\begin{array}{l}\text { Atlanta } \\
1996\end{array}$ & 197 & 95 & $32,5 \%$ & 10 & 6 & $37,5 \%$ \\
\hline $\begin{array}{l}\text { Sidney } \\
2000\end{array}$ & 219 & 105 & $32,4 \%$ & 7 & 4 & $36,4 \%$ \\
\hline $\begin{array}{l}\text { Atenas } \\
2004\end{array}$ & 180 & 140 & $43,8 \%$ & 13 & 4 & $23,5 \%$ \\
\hline $\begin{array}{l}\text { Pekín } \\
2008\end{array}$ & 164 & 122 & $42,7 \%$ & 12 & 5 & $29,4 \%$ \\
\hline $\begin{array}{l}\text { Londre } \\
\text { s } 2012\end{array}$ & 169 & 112 & $39,9 \%$ & 6 & 12 & $66,7 \%$ \\
\hline Río & 165 & 145 & $46,8 \%$ & 8 & 9 & $52,9 \%$ \\
\hline
\end{tabular}

Fuente: www.coe.es (Comité Olímpico Español).

Esta tendencia hacia la reducción de la brecha de género en la práctica deportiva popular, el importante incremento de la práctica deportiva femenina de alto nivel y la demostración del alto rendimiento deportivo de las mujeres no tiene, sin embargo, reflejo en el trato mediático que reciben las mujeres deportistas. La invisibilidad de las mujeres deportistas y su representación estereotipada es una realidad testaruda. Éstas están infrarrepresentadas en los medios de comunicación, en la prensa generalista y en la prensa deportiva especializada, tanto en papel como digital. Diferentes estudios (Rintala y Birrell, 1984; Duncan y Messner, 1990, 1991, 1994, 2000; Pearson, Turner y Mancillas, 1993; Ibañez y Lacosta, 1998; Flink y
Kensicki, 2002; y Sainz de Baranda, 2013, 2014; GMMP, 2015; Matud y Rodríguez, 2017) muestran esta desigualdad de presencia mediática entre mujeres y hombres deportistas. Pero, además, se observa que tanto ellas como ellos son representados según los estereotipos de género más tradicionales, atribuyendo poder y autoridad a los hombres y belleza a las mujeres, además de "cosificar" a las mujeres deportistas y visibilizarlas con algún tipo de connotación sexual. En esta línea de trabajo podrían citarse estudios como Fink y Kensicki (2002), RojasTorrijos (2010), Marín y Ganzabal (2011), Sainz de Baranda (2014), GMMP (2015) o Rangel y Ramírez (2018), entre otros muchos.

La representación mediática juega un papel crucial en la construcción de la opinión pública. Tal como señala Lippman (2003), la imagen mental que construye la población de los hechos es una construcción imaginaria de sucesos a los que no tiene acceso inmediato ni real. Y, si bien no existe consenso sobre el nivel de influencia y el alcance, los estudios constatan que los medios de comunicación tienen efectos palpables en la opinión pública (Price, 1994; McQuail, 2000; Wolf, 2001; Roiz, 2005).

Todo esto hace que sea pertinente estudiar cuál es la opinión de la ciudadanía sobre las mujeres deportistas, determinar si la ciudadanía conoce a las mujeres deportistas tanto como a los hombres deportistas y descubrir si existe o no una imagen estereotipada y sesgada de las mujeres deportistas. Por ello, el propósito general de esta investigación es determinar la percepción que tiene la ciudadanía guipuzcoana de la figura de las mujeres deportistas en comparación con la de los hombres deportistas. Este objetivo general se despliega en los siguientes objetivos específicos de carácter (a) cognoscitivo, (b) afectivo y (c) conativo o de comportamiento:

- Sobre la visibilidad de las mujeres deportistas. En el nivel cognoscitivo, se trata de estudiar:

La visibilidad de las mujeres deportistas en España en contraste con la de los hombres deportistas entre la población guipuzcoana.

La notoriedad de las mujeres deportistas de España en contraste con la de los hombres deportistas entre la población guipuzcoana.

- Sobre la imagen de las mujeres deportistas. A nivel afectivo, se plantean dos cuestiones: 


\section{Mujika-Alberdi, A.; García-Arrizabalaga, I.; Gibaja-Martíns, J. J.}

Determinar la importancia que tiene para la población guipuzcoana el aspecto físico en mujeres deportistas en contraste con la que tiene en hombres deportistas.

Identificar qué valores asocia la población guipuzcoana a las mujeres deportistas en contraste con los asociados a los hombres deportistas.

- Sobre el consumo de competiciones deportivas femeninas. A nivel conativo, se trata de conocer la intención conductual de la población guipuzcoana en cuanto a sus preferencias de consumo de competiciones deportivas de mujeres y de hombres.

\section{MATERIAL Y MÉTODOS}

\section{Diseño de investigación}

Esta investigación es un estudio descriptivo de carácter diacrónico y desarrollado a través de una encuesta de opinión. El cuestionario empleado fue diseñado ad hoc para este estudio por los autores. Se trata de un cuestionario estructurado con cuestiones que tratan de responder a cada uno de los objetivos específicos detallados previamente. En concreto, el cuestionario mide:

- La visibilidad de las mujeres deportistas. La visibilidad se refiere a la percepción que tienen los ciudadanos de la presencia o no de las mujeres deportistas en la sociedad. Se entiende que la visibilidad es previa a la notoriedad y que sin visibilidad no cabe notoriedad. Se pidió puntuar la visibilidad actual de la mujer y del hombre deportista en una escala de 0 a 10 , donde 0 era nada visible y 10 totalmente visible. También se preguntó por la visibilidad de la mujer y del hombre deportista hace 5 años. Asimismo, para cada persona encuestada se calcularon las diferencias de visibilidad entre mujeres y hombres deportistas hoy y hace cinco años, y también la variación de la visibilidad de las mujeres y los hombres deportistas hoy y hace cinco años.

- La notoriedad de las mujeres deportistas. La notoriedad se refiere al grado de conocimiento que tienen los encuestados sobre los deportistas. Se midió tanto la notoriedad espontánea como la asistida. Para la notoriedad espontánea se pidió a los encuestados que nombraran hasta cinco figuras de deportistas de renombre que conociesen. Para la notoriedad asistida se diseñó una lista de 20 deportistas, mujeres y hombres, a partir del ranking elaborado por el diario deportivo de mayor difusión $\mathrm{y}$ audiencia de España, Marca (www.marca.com ${ }^{1}$ ). Se creó un índice de notoriedad asistida en base a los siguientes criterios: se asignó el valor de 1 si la persona encuestada afirmaba conocer al o a la deportista e identificaba correctamente el deporte practicado; un valor de ,25 si afirmaba conocer al o a la deportista, pero no recordaba el deporte que practicaba; un valor de, $15 \mathrm{si}$ afirmaba conocer al o a la deportista, pero cometía un error al asociar el deporte practicado; y un valor de 0 si no conocía al o a la deportista.

- Para conocer la importancia del aspecto físico de mujeres y hombres deportistas se emplearon tres ítems. El primero, enunciado de manera directa, "La belleza física de las personas que se dedican al deporte profesional es importante". El segundo, donde se planteaba si "La belleza física es más importante en el caso de las mujeres deportistas profesionales que en el caso de los hombres deportistas profesionales". El último, enunciado de manera indirecta con objeto de evitar sesgo por deseabilidad social, "La gente, en general, se fija más en la belleza física de las mujeres deportistas profesionales que en la de los hombres deportistas profesionales". En los tres ítems se utilizó una escala Likert de cinco posiciones que iba desde totalmente en desacuerdo (con un valor asignado de -2) hasta totalmente de acuerdo (con un valor asignado de +2 ).

- Con objeto de conocer la masculinidad o feminidad de los valores asociados al deporte, se seleccionó una batería de valores (véase la primera columna de la Tabla 10) y se midió la percepción en una escala de cinco posiciones de respuesta, desde -2 ("Ese valor lo asocio

\footnotetext{
${ }^{1}$ Marca: El ránking definitivo de los 30 mejores deportistas de la historia. https://www.marca.com/otrosdeportes/2018/06/12/5b1ebf31e2704e39168b45f1.html
} 


\section{Visibilidad social de las mujeres deportistas}

totalmente a los hombres") hasta +2 ("Ese valor lo asocio totalmente a las mujeres"). Una respuesta neutra indicaría que ese valor no se aprecia claramente vinculado a hombres o a mujeres.

- Para observar el comportamiento de las personas encuestadas se preguntaron varias cuestiones referidas a la preferencia a la hora de ver competiciones deportivas de mujeres y hombres. A las personas encuestadas se les preguntó, en una escala de 0 a 10, cuánto les gustaba ver una competición de categoría masculina y cuánto una de categoría femenina. A partir de estas dos cuestiones se calculó una tercera variable que es la diferencia de la valoración entre ver competiciones masculinas y femeninas. Siguiendo esta línea de preguntas, se consultó cuánto estarían dispuestos a pagar por un acontecimiento femenino comparado con lo que pagarían por uno masculino.

El cuestionario fue administrado de manera independiente al equipo de investigación por un instituto de estudios de mercado mediante encuestas telefónicas con sistema CATI.

\section{Participantes}

El método de muestreo fue el aleatorio con cuotas proporcionales de comarca de residencia, sexo y edad. La muestra final estaba compuesta por 406 guipuzcoanos/as mayores de edad. La ficha técnica de la encuesta realizada es la que se detalla en la Tabla 2.

Tabla 2. Ficha técnica de la encuesta

\begin{tabular}{|c|c|}
\hline $\begin{array}{l}\text { Diseño del } \\
\text { cuestionario }\end{array}$ & Equipo de investigación \\
\hline $\begin{array}{l}\text { Empresa que } \\
\text { realiza el trabajo } \\
\text { de campo }\end{array}$ & $\begin{array}{l}\text { Datakey (https://instituto- } \\
\text { datakey.com/) }\end{array}$ \\
\hline $\begin{array}{l}\text { Fecha del trabajo } \\
\text { de campo }\end{array}$ & 9 al 20 de diciembre de 2019 \\
\hline $\begin{array}{l}\text { Ámbito geográfico } \\
\text { del estudio }\end{array}$ & Gipuzkoa \\
\hline Unidad estadística & $\begin{array}{l}\text { Personas de } 18 \text { a } 80 \text { años } \\
\text { cumplidos }\end{array}$ \\
\hline $\begin{array}{l}\text { Tamaño del } \\
\text { universo }\end{array}$ & $\begin{array}{l}554.283 \text { personas ( } 274.778 \\
\text { hombres y } 279.505 \text { mujeres) }\end{array}$ \\
\hline $\begin{array}{l}\text { Método de } \\
\text { encuesta utilizado }\end{array}$ & $\begin{array}{l}\text { Cuestionario telefónico asistido } \\
\text { mediante sistema CATI }\end{array}$ \\
\hline
\end{tabular}

\begin{tabular}{ll}
\hline $\begin{array}{l}\text { Método de } \\
\text { muestreo }\end{array}$ & $\begin{array}{l}\text { Muestreo aleatorio (con cuotas de } \\
\text { comarca de residencia, sexo y } \\
\text { edad) }\end{array}$ \\
$\begin{array}{l}\text { Nivel de confianza } \\
\text { Estimación de la }\end{array}$ & $\begin{array}{l}\text { Se ha trabajado bajo condiciones } \\
\text { desfavorables de muestreo, } \\
\text { varianza }\end{array}$ \\
poblacional & $\begin{array}{l}\text { varianza poblacional }(\mathrm{P}=\mathrm{Q}=50 \%) \\
\text { Para las estimaciones globales, el } \\
\text { error máximo de muestro es del } \\
4,97 \%\end{array}$ \\
Error de muestreo & 406 encuestas válidas \\
& \\
\hline $\begin{array}{l}\text { Tamaño de la } \\
\text { muestra }\end{array}$ &
\end{tabular}

La Tabla 3 detalla la distribución de la muestra por edad, sexo y nivel de estudios de las personas encuestadas. La muestra se caracteriza por un mayor número de mujeres $(52,5 \%)$ que de hombres $(47,5 \%)$. La media de edad de las personas encuestadas se acerca a los 50 años con una desviación estándar de 16,41. Una prueba chi-cuadrado de comparación de proporciones por categorías de género, edad y comarca de residencia entre las distribuciones muestrales y poblacionales no arrojó resultados significativos, corroborando así que las cuotas muestrales de género, edad y comarca de residencia se ajustan a las cuotas poblacionales.

Tabla 3. Descripción de la muestra

\begin{tabular}{llcc}
\hline Variable & Categoría & $\mathbf{n = 4 0 6}$ & $\mathbf{\%}$ \\
\hline Sexo & Hombre & 193 & 47,5 \\
& Mujer & 213 & 52,5 \\
Edad & 18 a 35 & 89 & 21,9 \\
& 36 a 50 & 121 & 29,8 \\
& 51 a 65 & 111 & 27,3 \\
& 66 o más & 85 & 21,0 \\
\hline
\end{tabular}

Para el análisis de datos se han aplicado, por una parte, el análisis descriptivo univariante y, por otra, métodos paramétricos para la comparación de dos medias. La comparación entre grupos se realiza respecto a una variable continua y las observaciones comparadas se refieren a individuos de dos grupos independientes. La hipótesis nula que se trata de constatar es que las puntuaciones medias de los grupos comparados son iguales y, en su caso, que la puntuación media es igual a cero. El valor $p$ será el que indique la probabilidad de que se verifique la hipótesis nula. Tomaremos 0,05 como el valor crítico de p para tal verificación. Los 


\section{Mujika-Alberdi, A.; García-Arrizabalaga, I.; Gibaja-Martíns, J. J.}

datos fueron procesados con los programas IBM SPSS Statistics versión 23 y R.

\section{RESULTADOS}

\section{Sobre la visibilidad de las mujeres deportistas}

Para el conjunto de la muestra, la puntuación media de la visibilidad de la mujer deportista en la actualidad fue de 5,66 ( $\sin$ diferencias significativas en la puntuación dada por mujeres y hombres, $\mathrm{p}=, 737$ ) cuando la del hombre deportista es de 9,01 (también sin diferencias significativas entre las submuestras, $\mathrm{p}=, 181$ ). La visibilidad de ambos, mujeres y hombres deportistas, aumentó respecto a hace cinco años. En el caso de las mujeres deportistas, la puntuación media de visibilidad hace cinco años para el conjunto de la muestra fue de 3,20 -lo que supone un incremento de 2,46 puntos en los últimos cinco años-, sin diferencias estadísticamente significativas entre las puntuaciones dadas por mujeres y hombres $(\mathrm{p}=, 083)$. En el caso de los hombres deportistas, la puntuación media de visibilidad hace cinco años para el conjunto de la muestra fue de 8,83 , lo que supone un incremento de ,18 puntos en un lustro En este caso tampoco se observan diferencias significativas entre mujeres y hombres, $\mathrm{p}=, 344$. (Véase Tabla 4).

Tabla 4. Visibilidad de la mujer y del hombre deportista (¿Cómo puntuaría, en una escala de 0 a 10...?)

\begin{tabular}{|c|c|c|c|c|c|c|c|}
\hline \multirow[t]{2}{*}{ Item } & \multicolumn{2}{|c|}{$\begin{array}{c}\text { Hombres } \\
(\mathbf{n}=193)\end{array}$} & \multicolumn{2}{|c|}{$\begin{array}{l}\text { Mujeres } \\
(\mathbf{n}=\mathbf{2 1 3})\end{array}$} & \multicolumn{2}{|c|}{$\begin{array}{c}\text { Total } \\
(\mathrm{n}=406)\end{array}$} & \multirow[t]{2}{*}{$\begin{array}{c}\mu_{h}=\mu_{m} \\
\mathbf{p}\end{array}$} \\
\hline & $\bar{x}_{h}$ & $s_{h}$ & $\bar{x}_{m}$ & $s_{m}$ & $\bar{x}$ & $s$ & \\
\hline $\begin{array}{l}\text { La visibilidad actual de } \\
\text { la MUJER deportista }\end{array}$ & 5,69 & 1,972 & 5,63 & 1,930 & 5,66 & 1,948 & ,737 \\
\hline $\begin{array}{l}\text { La visibilidad actual del } \\
\text { HOMBRE deportista }\end{array}$ & 8,92 & 1,104 & 9,08 & 1,315 & 9,01 & 1,220 & ,181 \\
\hline $\begin{array}{l}\text { La visibilidad de la } \\
\text { MUJER deportista hace } \\
5 \text { años }\end{array}$ & 3,38 & 2,015 & 3,04 & 1,943 & 3,20 & 1,983 & ,083 \\
\hline $\begin{array}{l}\text { La visibilidad del } \\
\text { HOMBRE deportista } \\
\text { hace } 5 \text { años }\end{array}$ & 8,76 & 1,235 & 8,89 & 1,509 & 8,83 & 1,385 & ,344 \\
\hline
\end{tabular}

En cuanto a la comparación de la visibilidad recogida en la Tabla 5 se extraen las siguientes ideas:

- La visibilidad actual de la mujer deportista fue menor que la del hombre deportista (ítem 1); lo fue para el conjunto de la muestra $(-3,35)$, para la submuestra de hombres $(-3,23)$ y la de mujeres ($3,46)$; además, lo fue en términos estadísticamente significativos $(\mathrm{p}=, 000)$ en todos los casos. También se observó que no hay diferencias significativas entre las puntuaciones dadas por hombres y mujeres $(\mathrm{p}=, 300)$.
- Hace cinco años, la visibilidad de la mujer deportista también era menor que la del hombre deportista (ítem 2); lo era para el conjunto de la muestra $(-5,63)$, para la submuestra de hombres ($5,38)$ y la de mujeres $(-5,85)$; y lo fue en términos estadísticamente significativos $(\mathrm{p}=, 000)$ en todos los casos. Y, tampoco se observaron diferencias significativas entre las puntuaciones dadas por hombres y mujeres $(\mathrm{p}=, 059)$.

- La visibilidad actual de la mujer deportista fue mayor que la de hace cinco años (ítem 3); y lo fue para el conjunto de la muestra $(2,46)$, para la submuestra de hombres $(2,31)$ y la de mujeres $(2,59)$; y lo fue en términos estadísticamente significativos $(p=, 000)$ en todos los casos. Además, en este caso, la puntuación media obtenida en la submuestra de las mujeres fue significativamente mayor que en la de los hombres $(\mathrm{p}=, 038)$. Esto es, el incremento de visibilidad de las mujeres deportistas en estos cinco últimos años fue significativamente mayor entre las mujeres encuestadas que entre los hombres encuestados.

- Por otro lado, la visibilidad actual del hombre deportista fue mayor que la de hace cinco años; y lo fue para el conjunto de la muestra $(, 18)$, para la submuestra de hombres $(, 16)$ y la de mujeres $(, 19)$; $\mathrm{y}$ lo fue en términos estadísticamente significativos $(p \leq, 002)$ en todos los casos. No se observaron diferencias significativas entre las puntuaciones dadas por mujeres y hombres $(\mathrm{p}=, 698)$.

Tabla 5. Comparaciones sobre la VISIBILIDAD de las personas deportistas

ITEM 1: Visibilidad actual de la MUJER deportista - Visibilidad actual del HOMBRE deportista ITEM 2: Visibilidad MUJER deportista hace 5 años - Visibilidad HOMBRE deportista hace 5 años ITEM 3: Visibilidad actual MUJER deportista - Visibilidad MUJER deportista hace 5 años ITEM 4: Visibilidad actual HOMBRE deportista - Visibilidad HOMBRE deportista hace 5 años $\bar{x}_{h} \quad s_{h} \quad s_{h} \quad \mu_{h}$

\begin{tabular}{ccccccccccc}
\multicolumn{10}{c}{$\mathrm{p}$} \\
\hline 1 & $-3,23$ & 2,31 &, 000 & $-3,46$ & 2,10 &, 000 & $-3,35$ & 2,20 &, 000 &, 300 \\
2 & $-5,38$ & 2,50 &, 000 & $-5,85$ & 2,51 &, 000 & $-5,63$ & 2,51 &, 000 &, 059 \\
3 & 2,31 & 1,21 &, 000 & 2,59 & 1,44 &, 000 & 2,46 & 1,34 &, 000 &, 038 \\
4 &, 16 &, 72 &, 002 &, 19 &, 91 &, 002 &, 18 &, 82 &, 000 &, 098 \\
\hline
\end{tabular}

Sobre la notoriedad de las mujeres deportistas

En la Tabla 6 se observan las diferentes combinaciones de número de mujeres y hombres deportistas mencionados. No todas las personas encuestadas llegaron a mencionar cinco deportistas (máximo permitido), pues no estaban obligadas a hacerlo y se refirieron a un número menor. Hay 40 individuos $(9,9 \%$ de las personas encuestadas) que llegaron a citar hasta cinco figuras de deportistas, 


\section{Visibilidad social de las mujeres deportistas}

siendo todos ellos deportistas hombres y no hay ningún encuestado que llegó a mencionar a cinco mujeres deportistas. En el otro extremo, se observa que 29 individuos (7,1\% de las personas encuestadas) no fueron capaces de citar a ningún hombre deportista y 344 individuos $(84,7 \%)$ no fueron capaces de citar a ninguna mujer deportista. En resumen, la Tabla 6 y la Figura 1 recogen visualmente cómo las mujeres deportistas fueron mencionadas en mucha menor medida que los hombres deportistas.

\section{Tabla 6}

Mención espontánea de mujeres y hombres deportistas profesionales.

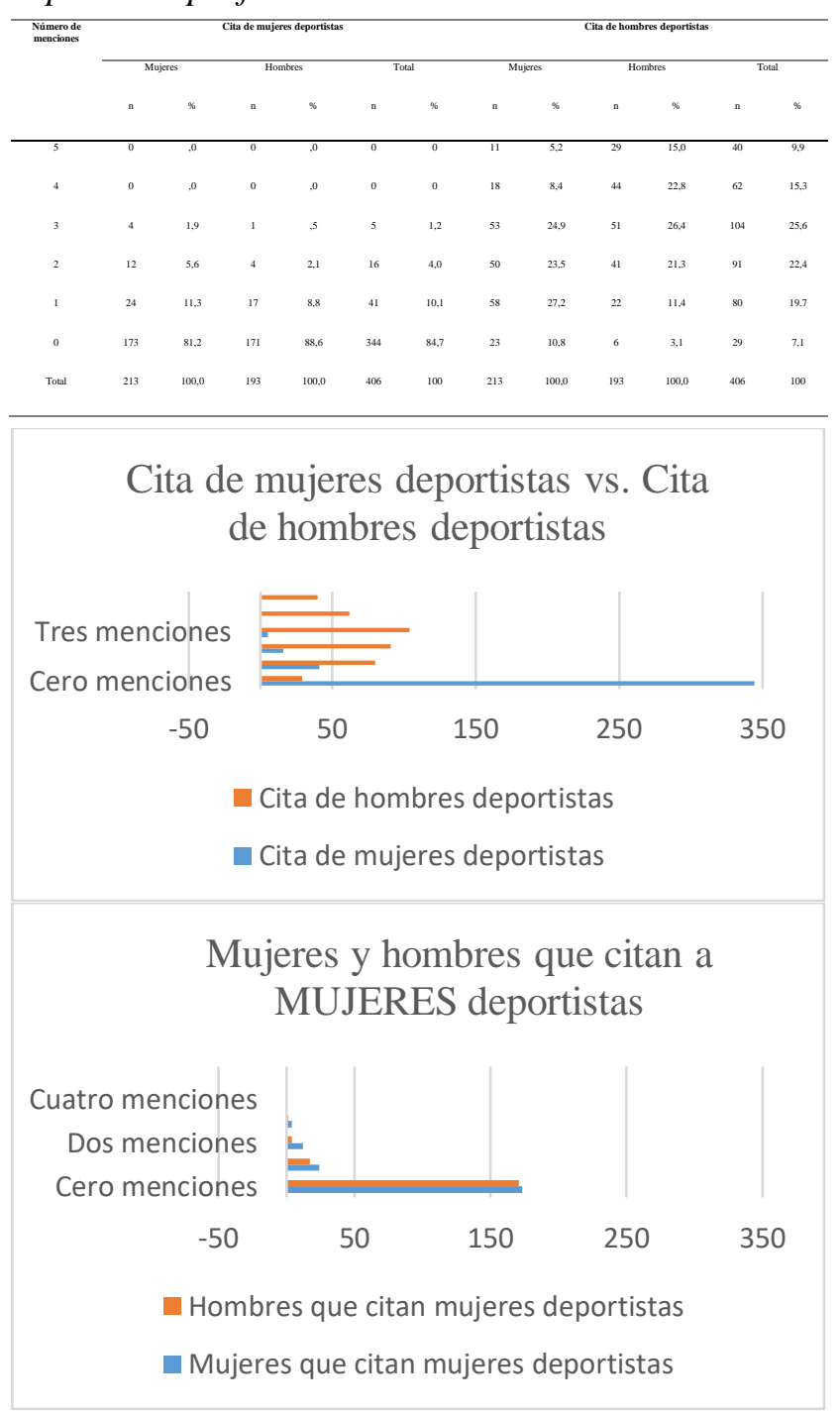

\section{Mujeres y hombres que citan a} HOMBRES deportistas

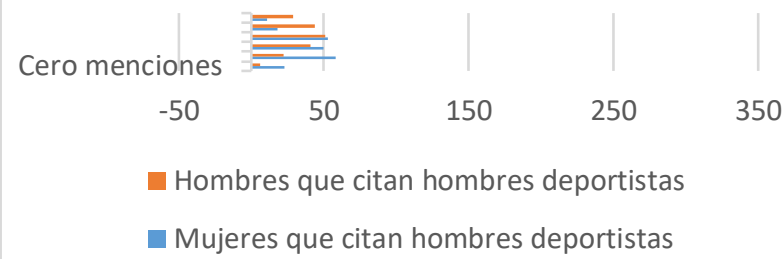

Figura 1. Mención espontánea de mujeres y hombres deportistas profesionales

Pasando ya al análisis individual de las mujeres y los hombres deportistas citados, el deportista con mayor número de menciones en primer lugar fue Rafa Nadal. El grupo de los cinco deportistas más mencionados lo completaron Leo Messi, Cristiano Ronaldo, Mikel Oyarzabal y Miguel Indurain. El ranking de los diez deportistas mencionados en segunda, tercera, cuarta o quinta posición no sufre grandes variaciones. De las 384 menciones en primer lugar, el $94,5 \%$ de ellas se refirieron a hombres deportistas y solo el 5,5\% a mujeres deportistas. Solo Edurne Pasaban obtuvo 4 menciones en primer lugar. En segunda mención, solo el 9,2\% de las mismas se refirieron a mujeres deportistas. Aparecía Mireia Belmonte en undécima posición con 6 menciones. En el resto de las menciones, se repitió el patrón de infra-visibilidad de las mujeres deportistas. Por último, los dos deportes más practicados por los deportistas que fueron mencionados por los encuestados fueron el fútbol y el tenis. Podemos concluir que el deporte se percibe en clave masculina y en torno a una red, la del tenis y la del fútbol.

Tabla 7. Notoriedad espontánea individual de mujeres y hombres deportistas.

\begin{tabular}{|c|c|c|c|c|c|c|c|c|c|c|}
\hline \multirow[t]{3}{*}{ Deportista } & \multicolumn{10}{|c|}{ Menciones } \\
\hline & \multicolumn{2}{|c|}{$\begin{array}{c}\text { Deportista } \\
\text { mencionado en } \\
\text { primer lugar }\end{array}$} & \multicolumn{2}{|c|}{$\begin{array}{c}\text { Deportista } \\
\text { mencionado en } \\
\text { segundo lugar }\end{array}$} & \multicolumn{2}{|c|}{$\begin{array}{c}\text { Deportista } \\
\text { mencionado en } \\
\text { tercer lugar }\end{array}$} & \multicolumn{2}{|c|}{$\begin{array}{c}\text { Deportista } \\
\text { Mencionado en } \\
\text { cuarto lugar }\end{array}$} & \multicolumn{2}{|c|}{$\begin{array}{l}\text { Deportista } \\
\text { mencionado } \\
\text { en quinto } \\
\text { lugar }\end{array}$} \\
\hline & $\mathrm{N}$ & $\%$ & $\mathrm{~N}$ & $\%$ & $\mathrm{~N}$ & $\%$ & $\mathrm{~N}$ & $\%$ & $\mathrm{~N}$ & \\
\hline Rafa Nadal & 125 & $32.6 \%$ & 21 & $6.9 \%$ & 29 & $12.9 \%$ & 13 & $10.2 \%$ & 3 & $4,3 \%$ \\
\hline Leo Messi & 61 & $15,9 \%$ & 53 & $17,4 \%$ & 25 & $11,2 \%$ & 5 & $3.9 \%$ & 5 & $7.2 \%$ \\
\hline Cristiano & 16 & $4,2 \%$ & 32 & $10,5 \%$ & 18 & $8,0 \%$ & 10 & $7,8 \%$ & 3 & $4,3 \%$ \\
\hline Ronaldo & & & & & & & & & & \\
\hline $\begin{array}{l}\text { Mikel } \\
\text { Oyarzabal }\end{array}$ & 14 & $3,6 \%$ & 10 & $3,3 \%$ & 4 & $1,8 \%$ & 4 & $3,1 \%$ & 0 &, $0 \%$ \\
\hline $\begin{array}{l}\text { Miguel } \\
\text { Indurain }\end{array}$ & 9 & $2,3 \%$ & 10 & $3,3 \%$ & 7 & $3,1 \%$ & 0 &, $0 \%$ & 0 &, $0 \%$ \\
\hline $\begin{array}{l}\text { Luis } \\
\text { Arconada }\end{array}$ & 8 & $2,1 \%$ & 4 & $1,3 \%$ & 3 & $1,3 \%$ & 0 &, $0 \%$ & 0 &, $0 \%$ \\
\hline Pau Gasol & 8 & $2,1 \%$ & 7 & $2,3 \%$ & 7 & $3,1 \%$ & 8 & $6,3 \%$ & 0 &, $0 \%$ \\
\hline $\begin{array}{l}\text { Fernando } \\
\text { Alonso }\end{array}$ & 6 & $1,6 \%$ & 12 & $3,9 \%$ & 7 & $3,1 \%$ & 0 &, $0 \%$ & 4 & $5,8 \%$ \\
\hline $\begin{array}{l}\text { Aimar } \\
\text { Olaizola }\end{array}$ & 6 & $1,6 \%$ & 0 & ,0\% & 4 & $1,8 \%$ & 0 &, $0 \%$ & 0 &, $0 \%$ \\
\hline $\begin{array}{l}\text { Jokin } \\
\text { Altuna }\end{array}$ & 5 & $1,3 \%$ & 6 & $2,0 \%$ & 4 & $1,8 \%$ & 3 & $2,3 \%$ & 0 &, $0 \%$ \\
\hline Xabi Prieto & 5 & $1,3 \%$ & 6 & $2,0 \%$ & 4 & $1,8 \%$ & 0 &, $0 \%$ & 0 &, $0 \%$ \\
\hline $\begin{array}{l}\text { Sergio } \\
\text { Ramos }\end{array}$ & 5 & $1,3 \%$ & 4 & $1,3 \%$ & 3 & $1,3 \%$ & 0 &, $0 \%$ & 0 &, $0 \%$ \\
\hline
\end{tabular}




\section{Mujika-Alberdi, A.; García-Arrizabalaga, I.; Gibaja-Martíns, J. J.}

\begin{tabular}{|c|c|c|c|c|c|c|c|c|c|c|}
\hline Xabi & 4 & $1,0 \%$ & 6 & $2,0 \%$ & 0 &, $0 \%$ & 3 & $2,3 \%$ & 0 &, $0 \%$ \\
\hline $\begin{array}{l}\text { Iker } \\
\text { Irribarria }\end{array}$ & 4 & $1,0 \%$ & 0 &, $0 \%$ & 0 &, $0 \%$ & 0 &, $0 \%$ & 0 &, $0 \%$ \\
\hline $\begin{array}{l}\text { Irribarria } \\
\text { Iñaki }\end{array}$ & 4 & $1,0 \%$ & 0 &, $0 \%$ & 0 & $.0 \%$ & 0 &, $0 \%$ & 0 &, $0 \%$ \\
\hline Artola & & & & & & & & & & \\
\hline $\begin{array}{l}\text { Edurne } \\
\text { pasaban }\end{array}$ & 4 & $1,0 \%$ & 0 &, $0 \%$ & 0 &, $0 \%$ & 0 &, $0 \%$ & 0 &, $0 \%$ \\
\hline $\begin{array}{l}\text { Iker } \\
\text { Casillas }\end{array}$ & 3 & $.8 \%$ & 0 &, $0 \%$ & 3 & $1,3 \%$ & 4 & $3,1 \%$ & 0 &, $0 \%$ \\
\hline $\begin{array}{l}\text { Marc } \\
\text { Marquez }\end{array}$ & 0 &, $0 \%$ & 8 & $2,6 \%$ & 0 &, $0 \%$ & 0 &, $0 \%$ & 0 &, $0 \%$ \\
\hline $\begin{array}{l}\text { Mireia } \\
\text { Belmonte }\end{array}$ & 0 &, $0 \%$ & 6 & $2,0 \%$ & 0 &, $0 \%$ & 4 & $3,1 \%$ & 0 &, $0 \%$ \\
\hline $\begin{array}{l}\text { Lebron } \\
\text { James }\end{array}$ & 0 &, $0 \%$ & 4 & $1,3 \%$ & 0 &, $0 \%$ & 0 &, $0 \%$ & 0 &, $0 \%$ \\
\hline $\begin{array}{l}\text { Aritz } \\
\text { Aduriz }\end{array}$ & 0 &, $0 \%$ & 4 & $1,3 \%$ & 0 &, $0 \%$ & 0 &, $0 \%$ & 0 &, $0 \%$ \\
\hline $\begin{array}{l}\text { Nahikari } \\
\text { García }\end{array}$ & 0 &, $0 \%$ & 4 & $1,3 \%$ & 0 &, $0 \%$ & 0 &, $0 \%$ & 0 &, $0 \%$ \\
\hline $\begin{array}{l}\text { David } \\
\text { Zurutuza }\end{array}$ & 0 &, $0 \%$ & 3 & $1,0 \%$ & 3 & $1,3 \%$ & 3 & $2,3 \%$ & 0 &, $0 \%$ \\
\hline $\begin{array}{l}\text { Antoine } \\
\text { Griezman }\end{array}$ & 0 &, $0 \%$ & 3 & $1,0 \%$ & 0 &, $0 \%$ & 0 &, $0 \%$ & 0 &, $0 \%$ \\
\hline $\begin{array}{l}\text { Aritz } \\
\text { Elustondo }\end{array}$ & 0 &, $0 \%$ & 3 & $1,0 \%$ & 0 &, $0 \%$ & 0 &, $0 \%$ & 0 &, $0 \%$ \\
\hline $\begin{array}{l}\text { Gerard } \\
\text { Piqué }\end{array}$ & 0 &, $0 \%$ & 3 & $1,0 \%$ & 0 &, $0 \%$ & 0 &, $0 \%$ & 0 &, $0 \%$ \\
\hline $\begin{array}{l}\text { Martín } \\
\text { Odegaard }\end{array}$ & 0 &, $0 \%$ & 3 & $1,0 \%$ & 0 &, $0 \%$ & 0 &, $0 \%$ & 0 &, $0 \%$ \\
\hline $\begin{array}{l}\text { Roger } \\
\text { Federer }\end{array}$ & 0 &, $0 \%$ & 0 &, $0 \%$ & 5 & $2,2 \%$ & 0 &, $0 \%$ & 0 &, $0 \%$ \\
\hline Jon Rahm & 0 &, $0 \%$ & 0 &, $0 \%$ & 4 & $1,8 \%$ & 0 &, $0 \%$ & 0 &, $0 \%$ \\
\hline $\begin{array}{l}\text { Oinatz } \\
\text { Bengoetxea }\end{array}$ & 0 &, $0 \%$ & 0 &, $0 \%$ & 3 & $1,3 \%$ & 0 &, $0 \%$ & 0 &, $0 \%$ \\
\hline Otros & 97 & $25,3 \%$ & 93 & $30,5 \%$ & 91 & $40,6 \%$ & 71 & $55,5 \%$ & 54 & $78,3 \%$ \\
\hline $\begin{array}{l}\text { Total } \\
\text { menciones }\end{array}$ & 384 & $100 \%$ & 305 & $100 \%$ & 224 & $100 \%$ & 128 & $100 \%$ & 69 & $100 \%$ \\
\hline \multirow{3}{*}{ Sexo } & \multicolumn{10}{|c|}{ Menciones } \\
\hline & \multicolumn{2}{|c|}{$\begin{array}{c}\text { Deportista } \\
\text { mencionado en } \\
\text { primer lugar }\end{array}$} & \multicolumn{2}{|c|}{$\begin{array}{c}\text { Deportista } \\
\text { mencionado en } \\
\text { segundo lugar }\end{array}$} & \multicolumn{2}{|c|}{$\begin{array}{c}\text { Deportista } \\
\text { mencionado en } \\
\text { tercer lugar }\end{array}$} & \multicolumn{2}{|c|}{$\begin{array}{c}\text { Deportista } \\
\text { Mencionado en } \\
\text { cuarto lugar }\end{array}$} & \multicolumn{2}{|c|}{$\begin{array}{c}\text { Deportista } \\
\text { mencionado } \\
\text { en quinto } \\
\text { lugar }\end{array}$} \\
\hline & $\mathrm{N}$ & $\%$ & $\mathrm{~N}$ & $\%$ & $\mathrm{~N}$ & $\%$ & $\mathrm{~N}$ & $\%$ & $\mathrm{~N}$ & $\%$ \\
\hline $\begin{array}{l}\begin{array}{l}\text { Hombres } \\
\text { deportistas }\end{array} \\
\end{array}$ & 363 & $94,5 \%$ & 277 & $90,8 \%$ & 209 & $93,3 \%$ & 114 & $89,1 \%$ & 59 & $85,5 \%$ \\
\hline $\begin{array}{l}\text { Mujeres } \\
\text { deportistas }\end{array}$ & 21 & $5,5 \%$ & 28 & $9,2 \%$ & 15 & $6,7 \%$ & 14 & $10,9 \%$ & 10 & $14,5 \%$ \\
\hline Total & \multirow{2}{*}{\multicolumn{10}{|c|}{ Menciones }} \\
\hline \multirow{3}{*}{$\begin{array}{l}\text { Deporte } \\
\text { practicado }\end{array}$} & & & & & & & & & & \\
\hline & \multicolumn{2}{|c|}{$\begin{array}{c}\text { Deportista } \\
\text { mencionado en } \\
\text { primer lugar }\end{array}$} & \multicolumn{2}{|c|}{$\begin{array}{c}\text { Deportista } \\
\text { mencionado en } \\
\text { segundo lugar }\end{array}$} & \multicolumn{2}{|c|}{$\begin{array}{c}\text { Deportista } \\
\text { mencionado en } \\
\text { tercer lugar }\end{array}$} & \multicolumn{2}{|c|}{$\begin{array}{c}\text { Deportista } \\
\text { Mencionado en } \\
\text { cuarto lugar }\end{array}$} & \multicolumn{2}{|c|}{$\begin{array}{c}\text { Deportista } \\
\text { mencionado } \\
\text { en quinto }\end{array}$} \\
\hline & $\mathrm{N}$ & $\%$ & $\mathrm{~N}$ & $\%$ & $\mathrm{~N}$ & $\%$ & $\mathrm{~N}$ & $\%$ & $\mathrm{~N}$ & $\%$ \\
\hline Fútbol & 140 & $36,5 \%$ & 166 & $54,4 \%$ & 95 & $42,4 \%$ & 47 & $36,7 \%$ & 21 & $30,4 \%$ \\
\hline Tenis & 136 & $35,4 \%$ & 33 & $10,8 \%$ & 39 & $17,4 \%$ & 20 & $15,6 \%$ & 10 & $14,5 \%$ \\
\hline Pelota & 25 & $6,5 \%$ & 16 & $5,2 \%$ & 17 & $7,6 \%$ & 8 & $6,3 \%$ & 6 & $8,7 \%$ \\
\hline Baloncesto & 17 & $4,4 \%$ & 25 & $8,2 \%$ & 17 & $7,6 \%$ & 15 & $11,7 \%$ & 5 & $7,2 \%$ \\
\hline Ciclism & 17 & $4,4 \%$ & 14 & $4,6 \%$ & 13 & $5,8 \%$ & 7 & $5,5 \%$ & 5 & $7,2 \%$ \\
\hline Atletismo & 13 & $3,4 \%$ & 9 & $3,0 \%$ & 7 & $3,1 \%$ & 7 & $5,5 \%$ & 6 & $8,7 \%$ \\
\hline Motor & 12 & $3,1 \%$ & 22 & $7,2 \%$ & 13 & $5,8 \%$ & 8 & $6,3 \%$ & 6 & $8,7 \%$ \\
\hline Montaña & 6 & $1,6 \%$ & 1 & $3 \%$ & 1 &, $4 \%$ & 2 & $1,6 \%$ & 2 & $2,9 \%$ \\
\hline Golf & 5 & $1,3 \%$ & 4 & $1,3 \%$ & 6 & $2,7 \%$ & 6 & $4,7 \%$ & 3 & $4,3 \%$ \\
\hline Remo & 5 & $1,3 \%$ & 3 & $1,0 \%$ & 1 &, $4 \%$ & 0 &, $0 \%$ & 1 & $1,4 \%$ \\
\hline Balonmano & 2 &, $5 \%$ & 1 & $3 \%$ & 3 & $1,3 \%$ & 2 & $1,6 \%$ & 0 &, $0 \%$ \\
\hline Halterofilia & 1 &, $3 \%$ & 0 &, $0 \%$ & 0 &, $0 \%$ & 0 &, $0 \%$ & 0 &, $0 \%$ \\
\hline Natación & 1 &, $3 \%$ & 2 &, $7 \%$ & 2 & $.9 \%$ & 2 & $1,6 \%$ & 0 &, $0 \%$ \\
\hline Patinaje & 1 &, $3 \%$ & 2 &, $7 \%$ & 0 &, $0 \%$ & 2 & $1,6 \%$ & 0 & $0 \%$ \\
\hline Rugby & 1 &, $3 \%$ & 1 &, $3 \%$ & 3 & $1,3 \%$ & 0 &, $0 \%$ & 0 &, $0 \%$ \\
\hline Gimnasia & 0 &, $0 \%$ & 3 & $1,0 \%$ & 2 &, $9 \%$ & 1 & $8 \%$ & 1 & $1,4 \%$ \\
\hline Dep. Vasco & 0 &, $0 \%$ & 1 & $3 \%$ & 0 &, $0 \%$ & 0 &, $0 \%$ & 0 &, $0 \%$ \\
\hline $\mathrm{Sk}$ & 0 &, $0 \%$ & 0 & 0 & 3 & $1,3 \%$ & 1 & $8 \%$ & 1 & $1,4 \%$ \\
\hline Boxeo & 0 &, $0 \%$ & 0 &, $0 \%$ & 1 &, $4 \%$ & 0 &, $0 \%$ & 1 & $1,4 \%$ \\
\hline Billar & 0 &, $0 \%$ & 0 &, $0 \%$ & 0 &, $0 \%$ & 0 &, $0 \%$ & 0 &, $0 \%$ \\
\hline Otros & 2 & $.5 \%$ & 2 &, $7 \%$ & 1 & $4 \%$ & 0 &, $0 \%$ & 1 & $1,4 \%$ \\
\hline Total & 384 & $100 \%$ & 305 & $100 \%$ & 224 & $100 \%$ & 128 & $100 \%$ & 69 & $100 \%$ \\
\hline
\end{tabular}

La Tabla 8 recoge los resultados de la notoriedad asistida de mujeres y hombres deportistas. El deportista con mayor notoriedad asistida resultó ser Iker Casillas, que, junto a Rafa Nadal, Andrés Iniesta, Miguel Indurain, Fernando Alonso, Pau Gasol, Ángel Nieto, Arantxa Sánchez Vicario, Marc Márquez y Severiano Ballesteros formaron el grupo de los diez deportistas más conocidos. En este top 10 solo aparecía una mujer y lo hacía en octava posición. El resto de las mujeres aparecían copando las últimas posiciones del ranking.
Tabla 8. Notoriedad asistida de mujeres y hombres deportistas

\begin{tabular}{|c|c|c|c|c|c|c|c|c|c|c|}
\hline \multirow[t]{2}{*}{$\begin{array}{l}\text { Figura } \\
\text { deportista }\end{array}$} & \multicolumn{2}{|c|}{$\begin{array}{c}\text { Conoce } \\
\text { deportista y } \\
\text { su deporte } \\
\text { (valor 1) }\end{array}$} & \multicolumn{2}{|c|}{$\begin{array}{c}\text { Conoce al } \\
\text { deportista, } \\
\text { pero no } \\
\text { recuerda su } \\
\text { deporte } \\
\text { (valor , 25) }\end{array}$} & \multicolumn{2}{|c|}{$\begin{array}{c}\text { Conoce al } \\
\text { deportista, } \\
\text { pero } \\
\text { comete } \\
\text { error al } \\
\text { asociar } \\
\text { deporte } \\
\text { (valor ,15) } \\
\end{array}$} & \multicolumn{2}{|c|}{$\begin{array}{c}\text { No conoce al } \\
\text { deportista } \\
\text { (valor 0) }\end{array}$} & \multicolumn{2}{|c|}{$\begin{array}{c}\text { Indice de } \\
\text { notoriedad del } \\
\text { deportista (en una } \\
\text { escala sobre 100) }\end{array}$} \\
\hline & $\mathrm{N}$ & $\%$ & $\mathrm{~N}$ & $\%$ & $\mathrm{~N}$ & $\%$ & $\mathrm{~N}$ & $\%$ & Media & $\begin{array}{c}\text { Desv. } \\
\text { Estandar }\end{array}$ \\
\hline Iker Casillas & 401 & 98,8 & 1 &, 2 & 0 &, 0 & 4 & 1,0 & 98,83 & 10,549 \\
\hline $\mathrm{RafaNaC}$ & 395 & 97,3 & 3 &, 7 & &, 5 & 6 & 1,5 & 97,55 & 14,815 \\
\hline $\begin{array}{l}\text { Miguel } \\
\text { Indurain }\end{array}$ & 385 & 94,8 & 3 &, 7 & 1 & 2 & 17 & 4,2 & 94,56 & 22,337 \\
\hline $\begin{array}{l}\text { Andrés } \\
\text { Iniesta }\end{array}$ & 383 & 94,3 & 3 &, 7 & 1 & , 2 & 19 & 4,7 & 94,56 & 22,337 \\
\hline $\begin{array}{l}\text { Fernando } \\
\text { Alonso }\end{array}$ & 375 & 92,4 & 5 & 1,2 & 14 & 3,4 & 12 & 3,0 & 93,19 & 23,854 \\
\hline Pau Gasol & 361 & 88,9 & 8 & 2,0 & 6 & 1,5 & 33 & 7,6 & 89,63 & 29,594 \\
\hline Ángel Nieto & 352 & 86,7 & $\begin{array}{l}6 \\
5\end{array}$ & 1,5 & 9 & 2,2 & 38 & $\begin{array}{l}6 \\
4\end{array}$ & $\begin{array}{l}87,4 \\
87,0\end{array}$ & $\begin{array}{l}32,369 \\
32712\end{array}$ \\
\hline $\begin{array}{l}\text { Arantxa } \\
\text { Sánchez } \\
\text { Vicario }\end{array}$ & 350 & 86,2 & 5 & 1,2 & 13 & 3,2 & 38 & 4 & 87,0 & 32,712 \\
\hline $\begin{array}{l}\text { Marc } \\
\text { Márquez }\end{array}$ & 329 & 81,0 & 13 & 3,2 & 10 & 2,5 & 54 & 13,3 & 82,2 & 37,079 \\
\hline $\begin{array}{l}\text { Severiano } \\
\text { Ballesteros }\end{array}$ & 326 & 80,3 & 17 & 4,2 & 15 & 3,7 & 48 & 11,8 & 81,9 & 36,883 \\
\hline $\begin{array}{l}\text { Xavi } \\
\text { Hernández }\end{array}$ & 303 & 74,6 & 12 & 3,0 & 5 & 1,2 & 86 & 21,2 & 75,55 & 42,193 \\
\hline $\begin{array}{l}\text { Garbiñ } \\
\text { Mugur }\end{array}$ & 296 & 72,9 & 24 & 5,9 & 19 & 4,7 & 67 & 16,5 & 75,09 & 41,284 \\
\hline $\begin{array}{l}\text { Conchita } \\
\text { Martínez }\end{array}$ & 277 & 68,2 & 16 & 3,9 & 8 & 2,0 & 105 & 25,9 & 69,51 & 45,005 \\
\hline $\begin{array}{l}\text { Mireia } \\
\text { Belmonte }\end{array}$ & 255 & 62,8 & 31 & 7,6 & 28 & 6,9 & 92 & 22,7 & 65,75 & 45,009 \\
\hline $\begin{array}{l}\text { Maialen } \\
\text { Chourraut }\end{array}$ & 195 & 48,0 & 20 & 4,9 & 28 & 6,9 & 163 & 40,1 & 50,3 & 48,222 \\
\hline $\begin{array}{l}\text { Carolin } \\
\text { Marín }\end{array}$ & 174 & 42,9 & 23 & 5,7 & 13 & 3,2 & 196 & 48,3 & 44,75 & 48,282 \\
\hline B Beitia & 16 & 40 & 3 & 9, & & 9 & 170 & & & 47,011 \\
\hline $\begin{array}{l}\text { Ona } \\
\text { Carbonell }\end{array}$ & 168 & 41,4 & 28 & 6 & 13 & 3,2 & 197 & 48,5 & 43,58 & 7,899 \\
\hline $\begin{array}{l}\text { Lydia } \\
\text { Valentín }\end{array}$ & 80 & 19,7 & 11 & 2,7 & 4 & 1,0 & 311 & 76,6 & 20,53 & 39,648 \\
\hline $\begin{array}{l}\text { Theresa } \\
\text { Zabell }\end{array}$ & 13 & 3,2 & 12 & 3,0 & 42 & 10,3 & 339 & 83,5 & 5,49 & 18,237 \\
\hline
\end{tabular}

Sobre la imagen de las mujeres deportistas. La importancia del aspecto físico de las mujeres deportistas

Como se puede apreciar en la Tabla 9, para el conjunto de la muestra, la puntuación media para "La belleza física de las personas que se dedican al deporte profesional es importante" fue de $-1,10$ $(\mathrm{p}=, 000)$, puntuación que es significativamente diferente a cero, tanto para la muestra en su conjunto como para mujeres y hombres considerados por separado. Además, las diferencias de opinión entre mujeres $(-1,17)$ y hombres $(-1,03)$ en este ítem no fueron estadísticamente significativas (véase la última columna, $\mathrm{p}=, 201$ ).

En el ítem "La belleza física es más importante en el caso de las mujeres deportistas profesionales que en el caso de los hombres deportistas profesionales" de nuevo la puntuación media para toda la muestra fue de $-1,10 \quad(\mathrm{p}=, 000)$, resultado que también es significativamente diferente de cero, tanto para la muestra en su conjunto como para mujeres y hombres considerados por separado. Al igual que en el caso anterior, las diferencias de opinión entre mujeres ($1,09)$ y hombres $(-1,12)$ en este ítem no fueron 


\section{Visibilidad social de las mujeres deportistas}

estadísticamente significativas (véase la última columna, $\mathrm{p}=$,777).

Como este segundo ítem podía llevar sesgo de deseabilidad social, se completó con el resultado del enunciado formulado en términos indirectos en el tercer ítem. En este caso la puntuación media para toda la muestra fue de, 20 , resultado que, una vez más, es significativamente diferente de cero, como lo es también para la muestra de mujeres y para la de hombres. Al igual que en los dos primeros ítems, tampoco se observaron diferencias significativas $(\mathrm{p}=, 703)$ entre las submuestras de mujeres $(, 19)$ y hombres $(, 22)$.

Tabla 9. Opinión sobre la importancia de la belleza física de las personas deportistas (desde $-2=$ "Totalmente en desacuerdo" hasta $+2=$ "Totalmente de acuerdo")

ITEM 1: La belleza física de las personas que se dedican al deporte profesional es importante.

ITEM 2: La belleza física es más importante en el caso de las mujeres deportistas profesionales que en el de

los hombres deportistas profesionales.
ITEM 3: La gente, en general, se fija más en la belleza física de las mujeres deportistas profesionales que en la \begin{tabular}{lllll}
\multicolumn{5}{l}{ de los hombres deportistas profesionales. } \\
\hline ITEM & HOMBRES $(\mathrm{n}=193)$ & MUJERES $(\mathrm{n}=213)$ & TOTAL $(\mathrm{n}=406)$ & $\mu_{h}=\mu_{m}$
\end{tabular}

\begin{tabular}{|c|c|c|c|c|c|c|c|c|c|c|}
\hline & $\underline{x}_{h}$ & $s_{h}$ & $\begin{array}{c}\mu_{h}=0 \\
\mathrm{p}\end{array}$ & $\underline{x}_{m}$ & $s_{m}$ & $\begin{array}{c}\mu_{m}= \\
0 \\
\mathrm{p}\end{array}$ & $\underline{x}$ & $s$ & $\begin{array}{c}\mu=0 \\
\mathrm{p}\end{array}$ & p \\
\hline 1 & $-1,03$ & 1,12 & ,000 & $\overline{1,17}$ & 1,14 & |000, & $-1,10$ & 1,13 & ,000 & 201 \\
\hline 2 & $-1,12$ & 1,02 & ,000 & & 1,10 & ,000 & $-1,10$ & 1,07 & ,000 &, 777 \\
\hline 3 & ,22 & 81 & ,000 &, 19 & 1,03 & ,009 & ,20 & ,93 & ,000 & , 703 \\
\hline
\end{tabular}

Sobre la imagen de las mujeres deportistas. Los valores asociados a las mujeres deportistas

En la Tabla 10 se muestran los valores en orden descendente de feminidad por el conjunto de de las personas encuestadas. El valor más ligado a las mujeres fue la flexibilidad $(, 80)$, a mucha distancia de los dos siguientes, la sincronización $(, 38)$ y la coordinación $(, 35)$. Por el contrario, los tres valores que de las personas encuestadas más relacionaron con los hombres, de mayor a menor, fueron fuerza $(-, 81)$, corrupción $(-, 67)$ y potencia $(-, 57)$. Precisión fue el valor "neutro" para el conjunto de la muestra $(, 00)$.

Este "ranking" de valores femeninos y masculinos muestra algunas diferencias cuando se analizan los resultados por las submuestras de mujeres y hombres. El valor de flexibilidad fue el considerado, de manera significativa, como más femenino tanto por los hombres $(, 86)$ como por las mujeres $(, 74)$. Sin embargo, las mujeres destacaron en segundo lugar la coordinación $(, 33)$ y los hombres la sincronización (,46), en ambos casos de manera significativa. El tercer valor destacado por las mujeres fue el sacrificio $(, 32)$ y los hombres la coordinación $(, 38)$, en ambos casos, de nuevo, de manera significativa. En cuanto a los valores deportivos asociados a los hombres, mujeres y hombres coincidieron de manera significativa en destacar como los principales los mismos tres valores y lo hacían en el mismo orden: fuerza, corrupción y potencia.

Los resultados también indican la existencia de valores deportivos neutros o que estadísticamente no están asociados ni a lo femenino ni a lo masculino (p>,05). En el bloque de columnas para la muestra total (columna 10), se observaron algunos valores que se percibían como neutros, esto es, no ofrecen una media significativamente distinta de cero y en los que, por tanto, la muestra total no apreciaba una masculinidad o feminidad significativa. Estos valores fueron Sentido de equipo $(p=, 078)$, "Aguante" $(\mathrm{p}=, 341)$, Compañerismo $\quad(\mathrm{p}=, 348), \quad$ Precisión $(\mathrm{p}=, 920)$, Carisma $(\mathrm{p}=, 284)$ y Frialdad $(\mathrm{p}=, 086)$. Así pues, en la lista de valores, hasta el valor Sentido de equipo, la muestra en su conjunto los percibía como significativamente femeninos, mientras que por debajo de Frialdad los percibía como significativamente masculinos.

Este resultado difiere si se analizan las submuestras de hombres y mujeres por separado. Para ellos, la submuestra de hombres, Sentido de equipo $(\mathrm{p}=, 425)$, "Aguante" $\quad(p=, 620), \quad$ Compañerismo $\quad(p=, 702)$, Precisión $(\mathrm{p}=, 378)$ y Frialdad $(\mathrm{p}=, 052)$ fueron los valores que se percibían como significativamente neutros o no vinculados a hombres o a mujeres (véase columna 4). Para las mujeres, estos valores neutros fueron Deportividad $(\mathrm{p}=, 208)$, Concentración $(\mathrm{p}=, 096)$, Estrategia ( $\mathrm{p}=, 438)$, Visión $(\mathrm{p}=, 493)$, Sentido de equipo $(\mathrm{p}=, 107)$, "Aguante" $(\mathrm{p}=, 076)$, Compañerismo $(\mathrm{p}=, 096)$, Precisión $(\mathrm{p}=, 337)$ y Carisma $(\mathrm{p}=, 295)$ (véase columna 7).

Adicionalmente, se observaron diferencias estadísticamente significativas $(p<, 05)$ entre la opinión de las submuestras de mujeres y hombres para algunos valores (véase columna 11). En concreto:

- La feminidad de los valores deportivos de Sincronización y Juego limpio fue significativamente mayor para los hombres que para las mujeres (,46 frente a ,31 y, 29 frente a , 08 , respectivamente).

- La feminidad del valor Sacrificio fue significativamente menor para los hombres que para las mujeres (,20 frente a ,32).

- La masculinidad de valores como Arrogancia y Potencia fue significativamente mayor para los 


\section{Mujika-Alberdi, A.; García-Arrizabalaga, I.; Gibaja-Martíns, J. J.}

hombres que para las mujeres (-,51 frente a -,37 y ,- 64 frente a,- 50 , respectivamente).

Asimismo, las mujeres percibían el valor de Frialdad como un valor significativamente masculino $(-, 18)$, y los hombres como un valor significativamente neutro (o, en todo caso, más bien femenino, ya que para ellos vale,+ 08$)$. Por el contrario, los hombres percibían el valor Carisma como un valor significativamente masculino $(-, 10)$ y las mujeres lo percibían como un valor significativamente neutro (o, en todo caso, más bien femenino, ya que para ellas vale,+ 04$)$.

Tabla 10. Feminidad/Masculinidad de valores asociados al deporte (desde -2 = "Totalmente masculino" hasta +2 = "Totalmente femenino").

\begin{tabular}{|c|c|c|c|c|c|c|c|c|c|c|}
\hline \multirow{2}{*}{$\begin{array}{c}\text { VALOR } \\
\text { (1) }\end{array}$} & \multicolumn{3}{|c|}{ HOMBRES (n=193) } & \multicolumn{3}{|c|}{ MUJERES (n=213) } & \multicolumn{3}{|c|}{ TOTAL $(\mathrm{n}=406)$} & \multirow{2}{*}{$\begin{array}{c}\mu_{h}=\mu_{m} \\
\mathbf{P} \\
\mathbf{( 1 1 )}\end{array}$} \\
\hline & $\frac{x_{h}}{(2)}$ & $\begin{array}{l}s_{h} \\
\text { (3) }\end{array}$ & $\begin{array}{c}\mu_{h}= \\
0 \\
\mathrm{P} \\
(4)\end{array}$ & $\frac{x_{m}}{(5)}$ & $\begin{array}{l}s_{m} \\
(6)\end{array}$ & $\begin{array}{c}\mu_{m}= \\
0 \\
\mathrm{P} \\
(7)\end{array}$ & $\frac{x}{(8)}$ & $\begin{array}{c}S \\
(9)\end{array}$ & $\begin{array}{c}\mu= \\
0 \\
\mathrm{P} \\
(10)\end{array}$ & \\
\hline Flexibilidad & 86 &, 71 & 000 &, 74 & ,67 & ,000 & 80 & ,69 & ,000 & ,085 \\
\hline Sincronización &, 46 & 63 & ,000 & ,31 & ,63 & ,000 &, 38 & 63 & ,000 & ,024 \\
\hline Coordinación &, 38 & 65 & ,000 &, 33 & ,62 & ,000 &, 35 & ,63 & ,000 & ,432 \\
\hline Elegancia &, 37 & ,65 & ,000 &, 26 &, 54 & ,000 &, 31 &, 59 & ,000 & ,066 \\
\hline Equilibrio &, 32 & 60 & ,000 &, 26 & ,63 & ,000 &, 29 & ,61 &, 000 &, 338 \\
\hline Sacrificio &, 20 &, 56 & ,000 &, 32 & ,62 & ,000 & .27 & .60 & .000 &, 038 \\
\hline Escucha &, 29 &, 58 & ,000 &, 23 & ,62 & ,000 & ,26 & 60 &, 000 &, 314 \\
\hline Respeto &, 26 &, 56 & ,000 &, 26 & ,60 & ,000 &, 26 &, 58 &, 000 & ,988 \\
\hline Ética &, 23 &, 55 & ,000 &, 23 & ,60 & ,000 &, 23 &, 58 &, 000 &, 891 \\
\hline Superación &, 22 &, 55 & ,000 &, 23 & ,62 & ,000 &, 23 &, 58 &, 000 &, 837 \\
\hline Justicia &, 18 & 63 & ,000 & ,24 & 65 & ,000 &, 21 & ,64 & ,000 &, 325 \\
\hline Perseverancia &, 18 &, 54 & ,000 &, 23 & ,61 & ,000 &, 21 &, 58 &, 000 &, 396 \\
\hline Generosidad &, 21 &, 55 & ,000 & ,18 & ,61 & ,000 &, 20 &, 58 &, 000 & ,611 \\
\hline Solidaridad &, 22 &, 56 & ,000 & ,19 & ,62 & ,000 & ,20 &, 59 & ,000 & ,670 \\
\hline Colaboración &, 17 & ,49 & ,000 & ,20 &, 50 & ,000 & ,19 & ,49 &, 000 &, 528 \\
\hline Constancia &, 18 &, 51 & ,000 & ,20 &, 59 & ,000 & ,19 &, 55 &, 000 &, 774 \\
\hline Juego limpio &, 29 &, 57 & ,000 & ,08 &, 55 & ,042 & 18 & .56 & ,000 & ,000 \\
\hline Inteligencia &, 15 & 49 & ,000 &, 20 & ,48 & ,000 & ,17 & ,49 & ,000 & ,282 \\
\hline Esfuerzo & ,09 & .59 & ,040 &, 15 & ,62 & , &, 12 & ,61 & , & .343 \\
\hline Trabajo & ,09 & ,41 & 003 &, 15 & 48 & ,000 &, 12 &, 45 &, 000 & ,159 \\
\hline Control & ,12 &, 52 & ,001 &, 07 & 49 & ,035 & ,10 &, 50 &, 000 & ,278 \\
\hline Deportividad & ,13 &, 50 & ,000 &, 04 & 49 & ,208 & ,09 &, 50 & ,001 & ,061 \\
\hline Entusiasmo & ,08 & ,48 & ,018 &, 08 & 47 & ,009 & ,08 & ,47 &, 000 &, 973 \\
\hline Concentración & ,08 &, 39 & ,003 &, 06 & ,49 & ,096 & ,07 &, 45 &, 002 &, 548 \\
\hline Estrategia & ,12 & ,61 & 008 & ,03 & ,62 & ,438 & ,07 & 62 & ,016 & ,159 \\
\hline Visión & ,07 & 44 & ,023 &, 02 &, 50 & 493 & ,05 & ,47 &, 046 & ,296 \\
\hline Sentido equipo & ,03 & ,45 & ,425 &, 06 &, 55 & 107 &, 04 &, 51 &, 078 & ,480 \\
\hline "Aguante" &,- 03 & ,73 & 620 &, 09 &, 73 & ,076 &, 03 &, 73 &, 341 &, 112 \\
\hline Compañerismo &,- 02 &, 56 & ,702 &, 06 &, 49 & ,096 &, 02 &, 53 &, 398 &, 171 \\
\hline Precisión & ,03 & ,49 & ,378 &,- 03 &, 50 & ,337 &, 00 & ,49 &, 920 & 193 \\
\hline Carisma &,- 10 & ,49 & ,005 &, 04 &, 52 & ,295 &,- 03 &, 51 &, 284 & 007 \\
\hline Frialdad & ,08 & ,59 & ,052 &,- 18 & ,65 & ,000 &,- 05 & ,63 & ,086 & ,000 \\
\hline Éxito &,- 29 &, 56 & ,000 &,- 35 &, 56 & ,000 &,- 32 & .56 &, 000 &, 303 \\
\hline Velocidad &,- 42 & ,70 & ,000 &,- 33 & ,66 & ,000 &,- 37 & ,68 &, 000 & ,152 \\
\hline Liderazgo &,- 37 & 63 & ,000 &,- 44 & ,65 & ,000 &,- 41 & ,64 & ,000 & ,249 \\
\hline Arrogancia &,- 51 & 65 & ,000 &,- 37 & ,67 & ,000 &,- 44 & ,66 & ,000 & ,030 \\
\hline
\end{tabular}

\begin{tabular}{lllllllllll}
\hline Potencia &,- 64 &, 63 &, 000 &,- 50 &, 65 &, 000 & -57 &, 64 &, 000 &, 028 \\
Corrupción &,- 72 &, 66 &, 000 &,- 62 &, 62 &, 000 &,- 67 &, 64 &, 000 &, 132 \\
Fuerza &,- 80 &, 69 &, 000 &,- 82 &, 70 &, 000 &,- 81 &, 69 &, 000 &, 784 \\
\hline
\end{tabular}

La Figura 2 visualiza gráficamente las consideraciones realizadas previamente.

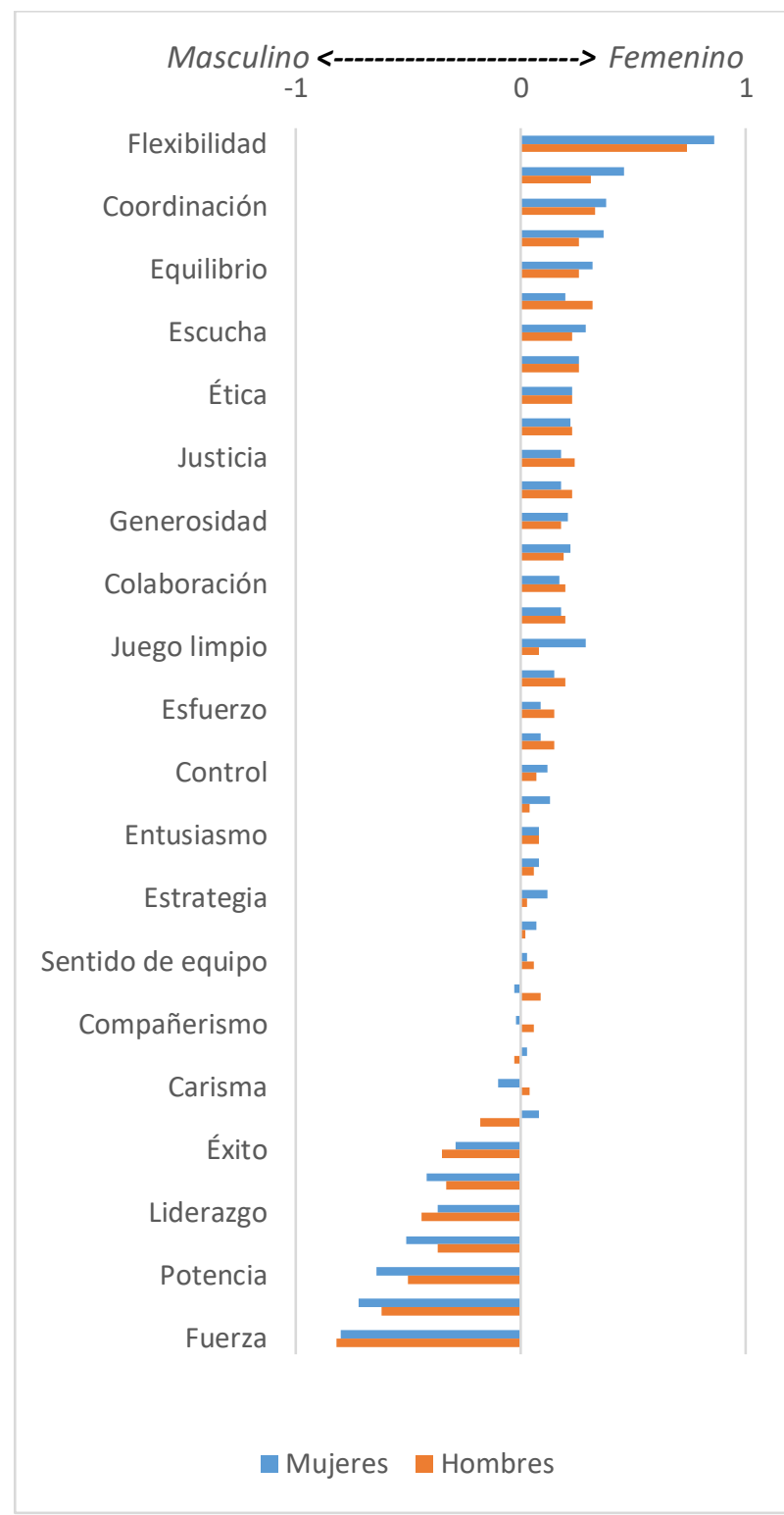

Figura 2. Feminidad/Masculinidad de valores asociados al deporte

Sobre el consumo de competiciones deportivas femeninas

La Tabla 11 recoge los resultados sobre preferencias en las competiciones deportivas de mujeres y hombres. Se observa que para el conjunto de la 


\section{Visibilidad social de las mujeres deportistas}

muestra apenas hay diferencias entre la primera y segunda cuestión. Esto es, por término medio los encuestados valoraban con 6,28 lo que les gusta ver una competición de categoría masculina y con 6,21 lo que les gustaba ver una de categoría femenina, apenas una diferencia de ,07 puntos. En el caso de la submuestra de hombres, esta diferencia fue mayor, alcanzando ,55 puntos $(6,91$ de preferencia por una competición masculina frente a 6,36 de una femenina), mientras, en el caso de la submuestra de mujeres, la diferencia fue negativa, esto es, decían que les gustaba ver más una competición de categoría femenina $(6,08)$ que una masculina $(5,70)$. También se observó que hay diferencias estadísticamente significativas entre mujeres y hombres $(p=, 000)$ para la variable "ver una competición de categoría masculina" y también para la nueva variable creada.

Siguiendo esta línea de preguntas, se consultó cuánto estarían dispuestos a pagar por un acontecimiento femenino comparado con lo que pagarían por uno masculino. Si fuera $100 €$ el precio a pagar por la asistencia a un acontecimiento deportivo masculino, las personas encuestadas decían estar dispuestas de promedio a pagar 94,91€ por uno femenino, aproximadamente un 5\% menos. En el caso de la submuestra de hombres, el precio a pagar por un acontecimiento femenino baja hasta los $88,33 €$, casi un $12 \%$ menos, y en el caso de la submuestra de mujeres decían que pagarían incluso algo más, $100,67 €$. Esta diferencia entre las respuestas de precio dadas por mujeres y hombres es estadísticamente significativa $(\mathrm{p}=, 000)$.

\section{Tabla 11}

Preferencias sobre las competiciones deportivas de mujeres y hombres.

\begin{tabular}{|c|c|c|c|c|c|c|c|c|}
\hline & \multicolumn{2}{|c|}{ Hombres } & \multicolumn{2}{|c|}{ Mujeres } & \multicolumn{2}{|c|}{ Total } & \multirow{2}{*}{$\begin{array}{c}\mathrm{t} \\
\text { Sudent }\end{array}$} & \multirow[t]{2}{*}{ p } \\
\hline & Media & $\begin{array}{l}\text { Desv. } \\
\text { Estandar }\end{array}$ & Media & $\begin{array}{l}\text { Desv. } \\
\text { Estandar }\end{array}$ & Media & $\begin{array}{l}\text { Desv. } \\
\text { Estandar }\end{array}$ & & \\
\hline $\begin{array}{l}\text { ¿Cuánto le gusta ver una } \\
\text { competición de categoria } \\
\text { masculina? }\end{array}$ & 6,91 & 2,577 & 5,70 & 3,002 & 6,28 & 2,869 & 4,360 &., 000 \\
\hline $\begin{array}{l}\text { ¿Cuánto le gusta ver una } \\
\text { competición de categoriá } \\
\text { femenina? }\end{array}$ & 6,36 & 2,533 & 6,08 & 3,071 & 6,21 & 2,828 & .997 & 319 \\
\hline $\begin{array}{l}\text { Diferencia de valoración } \\
\text { entre ver competiciones } \\
\text { masculinas y femenninas }\end{array}$ & .55 & 1,346 &,- 38 & 1,213 & ,07 & 1,358 & 7,286 & , 000 \\
\hline $\begin{array}{l}\text { Si usted pagara } 100 \text { e para } \\
\text { asistir a un acontecimiento } \\
\text { deportio masculino, } \\
\text { icuánto estaŕa dispuesto a } \\
\text { pagar por el mismo }\end{array}$ & 88,33 & 26,539 & 100,67 & 17,106 & 94,91 & 22,837 & $-5,398$ & , 000 \\
\hline
\end{tabular}

\section{DISCUSIÓN}

Los Juegos Olímpicos celebrados en Río el año 2016 se convirtieron en un imponente escenario donde las mujeres deportistas españolas pudieron demostrar su rendimiento. Casi la mitad de la expedición española estaba conformada por mujeres y fueron ellas quienes obtuvieron nueve de las diecisiete medallas. Además, de las siete medallas de oro de Rio 2016, cuatro fueron conseguidas por las deportistas féminas. Son datos que visibilizan el rendimiento de las mujeres en el deporte profesional cuando han tenido ocasión de demostrarlo. Además, los datos de la realidad social físicodeportiva son concluyentes, cada vez son más las mujeres que practican deporte y cada vez son más las que lo hacen de manera profesional (Encuesta de Hábitos Deportivos, 2015; Anuario de Estadísticas Deportivas, 2019; COE).

Sin embargo, esta realidad se encuentra con serias dificultades en la construcción de un imaginario social en cuanto al binomio práctica deportiva y mujeres. Las percepciones sociales se resumen en poca visibilidad y en mucho estereotipo. Es cierto que la visibilidad de las mujeres deportistas ha aumentado en los últimos cinco años, pero la diferencia existente entre las mujeres y los hombres deportistas sigue siendo extremadamente abultada, y las mujeres deportistas son todavía muy poco visibles. La medición de la notoriedad así lo ha evidenciado.

En notoriedad asistida, la primera mujer ocupaba la octava posición, la extenista Arantxa Sánchez Vicario. No parece ser casual, ya que los deportes practicados por los deportistas más notorios fueron fútbol y tenis. El deporte se percibe socialmente en clave masculina y, claramente, en torno a la "red" de una cancha de tenis y a la red de una portería de fútbol.

Además, la belleza física de las mujeres y hombres deportistas cambia de signo cuando se pregunta indirectamente a través de técnicas proyectivas. Todo indica que realmente la belleza física de las mujeres deportistas "pesa" para el público encuestado mucho más que la de los hombres deportistas y mucho más de lo que abiertamente se reconoce. Asimismo, se han observado diferencias sustanciosas en la asociación de determinados valores deportivos a mujeres y a hombres deportistas. La feminización de unos valores junto con la masculinización de otros sugiere una perpetuación de estereotipos de género que podría dar lugar a algunos obstáculos para alcanzar la paridad en el deporte de carácter competitivo, además de promover la percepción de la existencia de deportes femeninos y deportes masculinos. Las diferencias en la disposición a una conducta, en concreto, a la de consumir o no un evento y pagar más o menos por ello 


\section{Mujika-Alberdi, A.; García-Arrizabalaga, I.; Gibaja-Martíns, J. J.}

no hace más que subrayar la existencia de una brecha de género.

La escasa visibilidad y el exceso del estereotipo de las mujeres deportistas percibidos por la ciudadanía podrían ser combatidos de la mano de los medios de comunicación. Los medios de comunicación contribuyen a la formación de la opinión pública (Lippmann, 2003) y actúan como agentes de socialización mediando la experiencia individual (Ward y Harrison, 2005). Desafortunadamente, los datos sobre la visibilidad de las mujeres en general y de las deportistas en particular en los mass media no son reconfortantes.

El Proyecto de Monitoreo Global de Medios (Global Media Monitoring Project, conocido por su acrónimo, GMMP) puede considerarse como la primera investigación global que mide la presencia de las mujeres en los espacios informativos de mass media (radio, televisión y prensa escrita). En su primer informe, publicado en el año 1995, reveló que tan solo un $17 \%$ de los sujetos de las noticias eran mujeres. En el estudio publicado el año 2000 la cifra apenas sufrió modificaciones (18\%), en 2005 eran mujeres uno de cada cinco sujetos $(21 \%)$, en 2010 eran uno de cada cuatro (24\%) y, en el último informe, el de 2015, la proporción de mujeres alcanzaba la cifra del $28 \%$. En veinte años de observación se advierte que, aunque su presencia haya aumentado 11 puntos porcentuales, las mujeres siguen en una situación de invisibilidad relativa. Esta brecha de género se constata también en otros muchos estudios como Collins (2011), Matud et al (2011), Stanley (2012), Mateos de Cabo, et al (2014), Calvo y Gutiérrez (2016) o Matud y Rodríguez (2017).

El proyecto GMMP mide también la ocupación de las mujeres que son fuente de noticias. En el año 2015 en el 3\% de las ocasiones su ocupación es la de deportista, atleta, jugadora o entrenadora (GMMP, 2015) y en la mayoría de las ocasiones las mujeres están asociadas a cuestiones relacionadas con el cuidado y el ámbito privado y social. Por el contrario, este informe apunta que cuando son los hombres los sujetos de las noticias, aparecen en su gran mayoría relacionados entre otras cuestiones, con el deporte .

Otros estudios centrados en la información deportiva van en la misma dirección. Ibañez y Lacosta (1998) ya alertaron de la invisibilidad de las mujeres deportistas, conclusión que se repite sistemáticamente en estudios realizados por Rintala y Birrell (1984), Duncan y Messner (1990, 1991, 1994, 2000), Pearson, Turner y Mancillas (1993), Flink y Kensicki (2002) y Sainz de Baranda (2013 y 2014) o, más recientemente, Matud y Rodríguez (2017). Todos ellos repiten la idea de la infrarrepresentación de las mujeres deportistas.

Ciertamente, y como sostienen Alfaro et al (2011), la información deportiva se olvida de las mujeres y apenas las toma en consideración. Con el peligro de que, como sugiere López (2005), lo no visible corre el peligro de que deje de existir, de manera que, siguiendo a Calvo (2014), se perpetúa la visión androcéntrica del deporte. Hay incluso quien sostiene que la brecha de género en el deporte es una manera de representar las relaciones de poder y que el deporte en los medios está dominado por los hombres, realizado por ellos y para ellos (Crolley y Teso, 2007; Calvo y Gutiérrez, 2016).

Los estudios que se han centrado en analizar la presencia de mujeres y hombres deportistas en eventos muy específicos como los Juegos Olímpicos llegan también a la misma conclusión. En los Juegos Olímpicos de Río 2016, donde la participación de las mujeres superó el $45 \%$, los hombres eran noticia hasta tres veces más que las mujeres y, mientras los hombres deportistas eran noticia por sus cualidades físicas, las mujeres deportistas lo eran por su estado civil o edad (Borraz, 2016). Rangel y Ramírez (2018) añaden en su estudio, también centrado en los Juegos de Río 2016, que la referencia a la mujer deportista queda marginada a la parte final de la noticia, mientras que el hombre deportista se lleva el titular.

Según Rojas-Torrijos (2010), la información deportiva es el paradigma de los estereotipos sexistas. El GMMP (2015) observó que las mujeres en general son mostradas en los medios de comunicación en el ámbito doméstico o social y relacionadas con el cuidado familiar y el ámbito privado. Las mujeres deportistas en particular pocas veces aparecen en los medios por su valía, merecimiento y logros deportivos. Por el contrario, son mostradas como objetos sexuales, como madres y/o esposas (Fink y Kensicki, 2002), marginadas a la sombra de un hombre o como víctima (Marín y Ganzabal, 2011). Como sostiene Sainz de Baranda (2014), los éxitos deportivos de una mujer no aseguran su visibilidad y, en ocasiones, protagoniza noticias por actividades ajenas al deporte. 


\section{Visibilidad social de las mujeres deportistas}

Recientemente, Rangel y Ramírez (2018) inciden en el mismo sentido. En los hombres deportistas son noticia sus logros deportivos y en las mujeres deportistas son noticia diferentes aspectos de su vida privada, en particular, su maternidad.

En prensa digital se repiten los resultados. Mayoral y Mera (2017) analizaron la imagen de la mujer en los medios digitales deportivos y observaron que la presencia de la mujer deportista, además de ser insignificante, es habitual que aparezca con algún tipo de connotación sexual.

En otra línea de investigación, Martínez-Abajo, Vizcarra y Lasarte (2020) pusieron el foco no tanto en el estudio del contenido publicado en los mass media, sino en la percepción que las propias mujeres deportistas de élite tenían sobre el trato mediático recibido. En sus conclusiones recogen cómo estas mujeres deportistas se sienten invisibles, ninguneadas, tratadas como objetos y que los medios de comunicación no ponen en valor sus méritos deportivos. De esta manera es difícil que puedan convertirse en modelo para nadie y menos para las niñas.

En resumen, los mass media en general y el periodismo deportivo en particular coadyuvan en el mantenimiento de los estereotipos al reproducirlos sistemáticamente (Gómez-Collel, 2015), cuando, en realidad, su tarea, tal como recoge el Código Deontológico Europeo de la Profesión Periodística (Asamblea Parlamentaria del Consejo de Europa, 1993) debería ser la de reducir la brecha existente entre mujeres y hombres en el deporte. Una mayor presencia de las mujeres deportistas, acorde a su valía y merecimiento, las convertiría en referentes para otras mujeres y también para las niñas, visibilizándolas más allá de un cuerpo y de la belleza física.

\section{APLICACIONES PRÁCTICAS}

La realidad de la práctica deportiva de las mujeres no encuentra su equivalencia en la realidad social percibida por la ciudadanía. Apremia visibilizar a las mujeres deportistas y hacerlo sin estereotipos. Los medios de comunicación pueden jugar un papel relevante mostrando una imagen positiva de las deportistas, diversificada, exenta de estereotipos de género y como modelos de éxito personal, profesional y social. Un tratamiento informativo igualitario por los medios de comunicación en colaboración con el resto de instituciones sociales como la familia, o el sistema educativo favorecerán a alcanzar el reto de mayor visibilidad y menos estereotipo.

\section{LIMITACIONES}

El ámbito geográfico del trabajo de campo es el Territorio Histórico de Gipuzkoa y, si bien la muestra es representativa para ese marco geográfico, los resultados no deberían inferirse a otras poblaciones. En cualquier caso, los autores entienden que el estudio muestra una realidad de la desigualdad existente en el deporte que es de actualidad y de suma relevancia. Cabría también apuntar que la visibilidad de las mujeres deportistas se ha medido con un único ítem y no una escala, cuestión que merecería profundizar en futuros estudios. Asimismo, el estudio deja abierta otra línea de trabajo relacionada con la investigación motivacional sobre la actividad física y deportiva de sumo interés y relevancia.

\section{REFERENCIAS}

1. Alfaro, É., Álvarez, S., Barrero, J., Del Río, P., Escario, M., Fernández, C., Fontecha, M., Jiménez, E., López, G., Malvar, L., MoraFigueroa, B., Roldán, I., Sebastián, F. y Zamora, M. (2011). Deporte y mujeres en los medios de comunicación. Sugerencias y recomendaciones. CSD.

2. Asamblea Parlamentaria del Consejo de Europa (1993). Código Deontológico Europeo de la Profesión Periodística. Sitio. http://www.asociacionprensa.org/es/images/ Codigo_Deontologico_Europeo_de_la_Profe sion Periodistica.pdf

3. Borraz, M. (2016). Machismo, medios y deporte: ellos «fuertes»y ellas «solteras». Sitio.

http://www.eldiario.es/sociedad/MachismoJuegos-Olimpicos-fuertessolteras 0 546295460.html

4. Calvo Ortega, E. (2014). La representación de la mujer y los roles de Género en los informativos deportivos de televisión. Fonseca, Journal of Communication, 8(Enero-Junio de 2014), 111-129. 


\section{Mujika-Alberdi, A.; García-Arrizabalaga, I.; Gibaja-Martíns, J. J.}

5. Calvo, E. y Gutiérrez, B. (2016). Sexualidad, género, religión e interculturalidad en los relatos informativos civilizatorios y culturales de las televisiones españolas. Revista Latina de Comunicación Social, 71, 1090-1107.

6. COE [Comité Olímpico Español]. Sitio. http://www.coe.es/

7. Collins, R. L. (2011). Content analysis of gender roles in Media: We are we now and where should we go? Sex Roles, 64, 290-298.

8. Consejo Superior de Deportes (2015). Encuesta de Hábitos Deportivos en España. 2015. Ministerio de Cultura y Deporte. Sitio. http://www.culturaydeporte.gob.es/serviciosal-ciudadano/estadisticas/deportes/encuestahabitos-deportivos-en-espana.html

9. Consejo Superior de Deportes (2019). Anuario de Estadísticas Deportivas 2019. Ministerio de Cultura y Deporte.

10. Crolley, L. y Teso, E. (2007). Gendered narratives in Spain. International Review for the Sociology of Sport, 42, 149-166.

11. Duncan, M. C. (1990). Sports photographs and sexual difference: Images of men and women in the 1984 and 1988 Olympic Games. Sociology of Sport Journal,7, 22-43.

12. Duncan, M. C. y Messner, M. A. (1994). Gender stereotyping in televised sports: A follow up to the 1989 study. Estados Unidos: The Amateur Athletic Foundation of Los Angeles. Sitio: http://www.la84.org/genderstereotyping-in-televised-sports-a-follow-upto-the-1989-study

13. Duncan, M. C. y Messner, M. A. (2000). Gender in televised sports: 1989, 1993 and 1999. Estados Unidos: The Amateur Athletic Foun-dation of Los Angeles. Sitio: http://library.la84.org/9arr/ResearchReports/t v2000.pdf

14. Duncan, M. C., Messner, M. A. y Williams, L. (1991). Coverage of women's sports in four daily newspapers. Estados Unidos: The Amateur Athletic Foundation of Los Angeles. Sitio: http://www.la84.org/coverage-ofwomens-sports-in-four-daily-newspapers

15. Duncan, M.C. y Messner, M. (1994). Gender stereotyping in televised sports: A followup to the 1989 study. The Amateur Athletic Foundation of Los Angeles, Estados Unidos.
16. Fink, J. y Kensicki, L. (2002). An imperceptible difference: Visual and textual constructions of feminity in Sports Illustrated and Sports Illustrated for Women. Mass Commnication and Society, 5, 317-339. http://dx.doi.org/10.1207/S15327825MCS05 03_5

17. García, I., Gibaja, J. J. y Mujika, A. (2017). Opinión de la población guipuzcoana sobre la figura de las mujeres políticas. Anàlisi. Quaderns de Comunicació i Cultura, 56, 4970.

18. GMMP (2015). Proyecto de Monitoreo Global de Medios 2015 Informe Nacional.

19. Gómez-Collel, E. (2015). Adolescencia y deporte: ausencia de referentes femeninos en los medios para las adolescentes. Apunts. Educación Física y Deportes, $122\left(4 .^{\circ}\right.$ trimestre, octubre-diciembre), 81-87.

20. Ibáñez, M. E. y Lacosta, M. (1998). Informació esportiva: només per a ells. En J. Gallego, Gènere i informació. Barcelona: Institut Català de la Dona.

21. Lippmann, W. (2003). La opinión pública. San Lorenzo de El Escorial: Langre. España.

22. López, P. (2005). Representación de género en los medios informativos de radio $y$ televisión. Instituto Oficial de Radio y Televisión. Sitio. http:// www.pilarlopezdiez.eu/pdf/2InfReprGen_05. pdf

23. Marín, F. y Ganzabal, M. (2011). La mujer (in)visible: la construcción de la identidad femenina a través de la fotografía en El País y El Mundo.Enl@ce Revista Venezolana de Información, Tecnología y Conocimiento, 8(3), 51-67.

24. Martínez-Abajo, J., Vizcarra, M.T. y Lasarte, G. (2020). How do Sportswomen Perceive the Way they are Treated in the Media?. Apunts. Educación Física y Deportes, 139, 73-82. https://doi.org/10.5672/apunts.20140983.es.(2020/1).139.10

25. Mateos de Cabo, R., Gimeno, R., Martínez, M., López, L. (2014). Perpetuating gender inequality via the internet? An analysis of women's presence in Spanish online newspapers. Sex Roles, 70, 57-71.

26. Matud Aznar, M.P., Rodríguez-Wangüemert, C. y Espinosa Morales, I. (2017). 


\section{Visibilidad social de las mujeres deportistas}

Representación de mujeres y hombres en la prensa española. Revista Latina de Comunicación Social, 72, 765-782.

27. Matud, M. P., Rodríguez, C. y Espinosa, I. (2011). Gender in Spanish Daily Newspapers. Sex Roles, 64, 253-264.

28. Mayoral Sánchez, J. y Mera Fernández, M. (2017). La imagen de la mujer en la prensa deportiva digital: análisis de las portadas de as.com y marca.com. Cuadernos de Información y Comunicación, 22, 187-201.

29. McQuail, D. (2000). Introducción a la teoría de la comunicación de masas. Barcelona: Paidós.

30. Pearson, J.C., Turner, L.H. y Mancillas, W. T. (1993). Comunicación y género. Paidos. España.

31. Price, V. (1994). La opinión pública. Esfera pública y comunicación. Barcelona: Paidós.

32. Rangel A., del Carmen, G. y Ramírez Alvarado, M.M. (2018). Las mujeres en la prensa deportiva española durante los juegos olímpicos de Río 2016. Estudios sobre el Mensaje Periodístico, 24(2), 1595-1613.

33. Rintala, J. y Birrell, S. (1984). Fair treatment for the active female: a content analysis of Young Athlete magazine. Sociology of Sport Journal, 1(3), 231-250.

34. Roiz, M. (2005). Sociología de la comunicación y cultura de masas. Madrid: Ediciones del Laberinto.

35. Sainz de Baranda, C. (2014). Las mujeres en la prensa deportiva: dos perfiles. Cuadernos de Psicología del Deporte, 14(1), 91-102.
36. Sainz de Baranda, C. (2013). Mujeres y deporte en los medios de comunicación. Estudio de la prensa deportiva española (1979-2010) (Doctoral dissertation, Universidad Carlos III de Madrid).

37. Stanley, J. (2012). Women's absence from news photos: the role of tabloid strategies at elite and non-elite newspapers. Media, Culture \& Society, 34, 979-998.

38. Ward, L. M. y Harrison, K. (2005). The impact of media use on girls' beliefs about gender roles, their bodies, and sexual relationships: A research synthesis. En C. Ellen y J. Henderson (Eds.), Featuring females: Feminist analyses of media (pp. 323). Washington: American Psychological Association.

39. Wolf, M. (2001). Los efectos sociales de los media. Barcelona: Paidós. 